\title{
Experimental Investigation on the Radiation Pattern of a Horn Antenna Loaded by a Wire Medium
}

\author{
${ }^{1}$ Antônio Tomaz, ${ }^{2}$ Joaquim J. Barroso, ${ }^{2}$ P. J. Castro and ${ }^{1}$ Alberto J. Faro Orlando \\ ${ }^{1}$ Aeronautics Technological Institute (ITA), ${ }^{2}$ National Institute for Space Research (INPE) \\ São José dos Campos, SP, Brazil \\ attomaz@gmail.com
}

\begin{abstract}
The present paper reports on the design, implementation, and tests of a wire medium to experimentally verify its effects on the performance of a standard X-band horn antenna loaded by such a bulk metamaterial medium. The wire medium is composed of a periodic array of $0.5-\mathrm{mm}$-diameter metallic wires arranged in a square pattern with lattice spacing of $10.0 \mathrm{~mm}$ and hosted in Styrofoam plates. Experimental measurements of the radiation pattern at $11.70 \mathrm{GHz}$ show an enhancement of the side lobes while maintaining the antenna peak gain, indicating that the metamaterial wire medium introduces negligible loss into the loaded antenna.
\end{abstract}

\section{INTRODUCTION}

Wire medium, consisting of a periodic arrangement of metallic wires in a rectangular lattice, has been known since the 1950s in view of its applications as artificial dielectric with relative electric permittivity less than unity for microwave antenna lens in communications and radar system beamforming [1]. These arrays of wires behave as homogeneous bulk material whose electromagnetic properties can be described in terms of an effective permittivity. With the advent of metamaterials in the 2000s, renewed interest in wire media was sparked leading to a range of new applications such as subwavelength imaging in the visible spectrum, transport of near-field images, and directive emission [2]. The effects of incorporating a wire medium into horn antennas have been investigated in simulation [3] and experimental studies [4]-[6]. In [5], for instance, it has been demonstrated that placing a metamaterial wire liner on the inner wall of a C-band rectangular horn antenna has improved its performance over greater than an octave bandwidth. Related to these previous works which are concerned with ultra-refraction phenomena produced by wire media, the present paper reports on experiments to investigate the modifications on the radiation pattern of a standard X-band horn antenna by loading the antenna with a periodic arrangement of metallic wires especially designed to provide an average refraction index of 0.5 over the $8.0-12.0 \mathrm{GHz}$ frequency range.

\section{Model And Design of The Wire Medium}

The wire medium consists of thin metallic wires of diameter $d$ and arranged on a square lattice of a periodic spacing $a$, onto which impinges a TM electromagnetic wave with the electric field polarized along the direction $z$, as shown in Fig.1. Under this circumstance, and assuming lossless wires,

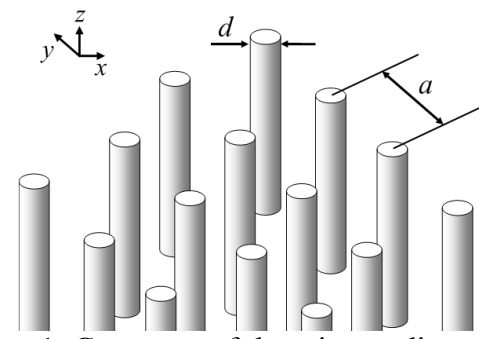

Figure 1. Geometry of the wire medium array.

the effective permittivity of the bulk homogenized medium exhibits a plasma-like dispersion expressed by

$$
\varepsilon=\varepsilon_{h}\left(1-\frac{f_{p}^{2}}{\varepsilon_{h} f^{2}}\right)
$$

where $\varepsilon_{\mathrm{h}}$ is the permittivity of the host medium, $f$ the frequency of the incident wave, and $f_{p}$ the equivalent plasma frequency. This important parameter is given by [2]

$$
f_{p}=c\left[a \sqrt{2 \pi\left(\ln \frac{a}{\pi d}+0.5275\right)}\right]^{-1}
$$

where $c$ the speed of light in free space.

To operate in the X-band the plasma frequency should be close to $8.0 \mathrm{GHz}$ so as to provide a near-zero permittivity at the lower edge of the $8.2-12.4 \mathrm{GHz}$ band. Then selecting the lattice spacing as $a=10.0 \mathrm{~mm}$ and the wire diameter $d=0.5 \mathrm{~mm}$ gives $f_{p}=7.7 \mathrm{GHz}$. The complete design and details of the characterization of wire medium are discussed in [7].

\section{IMPLEMENTATION OF THE WIRE MEDIUM AND ITS INCORPORATION INTO THE HORN ANTENNA}

For the realization of the experiment, a commercial horn antenna has been used [8] and then loaded with the wire medium. The arrays of metallic wires were accommodated and fixed in a Styrofoam plate properly machined in the bottom and top faces to match the flare angle of the antenna. This host material has a relative permittivity of 1.03 , with loss tangent of $0.1 \times 10^{-3}[9]$, thus being close to that of free space. Fig. 2 shows five layers of Styrofoam plates hosting the periodic array of metallic wires. Tests have been made using four wire-dielectric 
layers embedded into the antenna, for which the measured return loss is displayed in Fig. 3. Three pronounced dips appear in the return-loss spectrum at the frequencies of 7.9, 11.70, and 12.4 GHz. We note that the four layers used form three coupled resonators, whereby the original resonance frequency associated with the middle resonator is split into a band of three different frequencies, analogous to the Kroning-Penney potential wells in solid state physics [10].

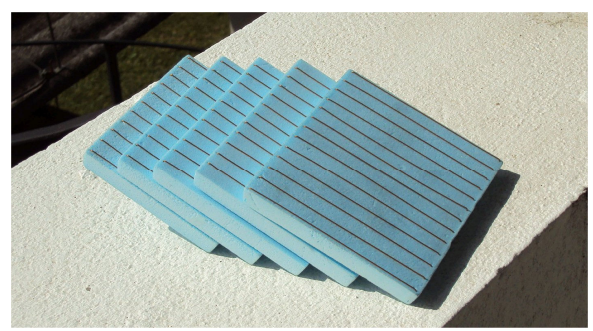

Figure 2. Copper wires fixed in the host layer.

Measurements of the radiation pattern have been performed with the antenna inside an anechoic chamber as shown in Fig. 4. Results are presented in Fig. 5, which compares the radiation patterns measured for the wire-medium loaded and empty antennas. The loading wire medium has the effect of enhancing the side lobes for both polarizations in experimental implementation. But we can see in Fig. 5(a) that the peak gain is maintained in the main lobe, indicating that the wire-dielectric layers introduce negligible loss in the horn. In addition, the cross-polarization level [Fig. 5(b)] is $20 \mathrm{~dB}$ lower than the corresponding co-polarization level for the loaded antenna.

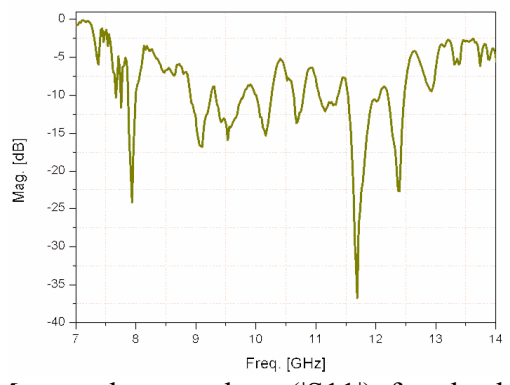

Figure 3. Measured return loss $(|\mathrm{S} 11|)$ for the horn antenna loaded with four composite layers.

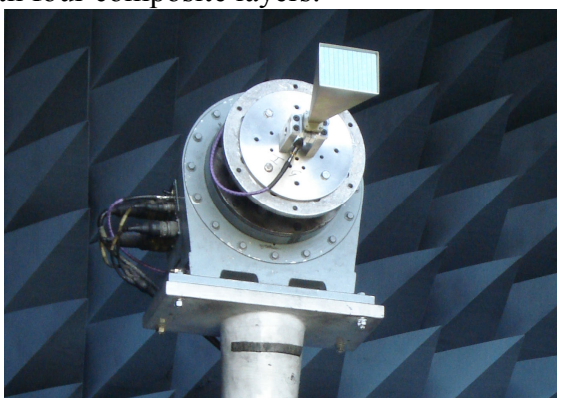

Figure 4. Antenna under test attached to the positioning system in the anechoic chamber.

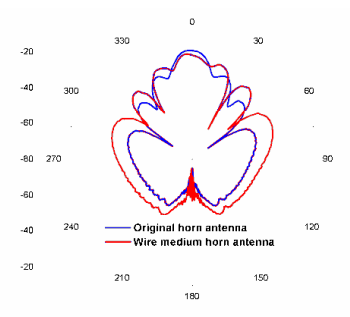

(a)

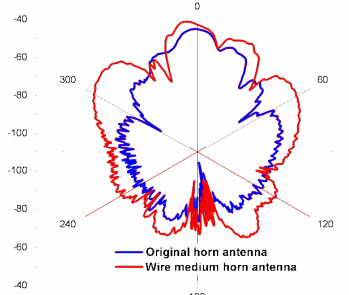

(b)
Figure 5. H-plane (a) co-polarized and (b) cross-polarized radiation patterns for the loaded and empty antennas measured at $11.70 \mathrm{GHz}$.

\section{CONCLUSION}

The present report has experimentally demonstrated the metamaterial wire medium capabilities to modify and control the radiation pattern of a standard horn antenna. Radiation patterns for the wire-medium-loaded antenna have been measured at $11.70 \mathrm{GHz}$, a frequency corresponding to a sharp resonance dip in the return loss spectrum of the meta antenna. Structural simplicity and low fabrication cost of the implemented wire medium enable the proposed arrangement to be used in further experiments aiming at enhancing directivity and gain of conventional microwave antennas.

\section{REFERENCES}

[1] Robert E. Collin, Field Theory of Guided Waves. New York: WileyIEEE Press, 1991.

[2] C. R. Simovski, P. A. Belov, A. V. Atrashchenko, and Y. S. Kivshar, "Wire metamaterials: physics and applications", Adv. Mater., vol. 24, pp. 4229-4248, Aug. 2012.

[3] Q. Wu, P. Pan, F.-Y. Meng, L.-W. Li and J.Wu, "A Novel Flat Lens Horn Antenna Designed Based on Zero Refraction Principle of Metamaterials,". Appl. Phys. A, vol. 87, pp. 151-156, 2007

[4] S. Hrabar, "Application of Wire Media in Antenna Technology," in S. Zouhdi, A. Sihvola, and A. P. Vinogradov, Metamaterials and Plasmonics: Fundamentals, Modelling, Applications (eds.), New York, Springer Netherlands, pp. 139-151, 2009.

[5] E. Lier, D. H. Werner, C. P. Scarborough, Q. Wu and J. A. Bossard, "An Octave Bandwidth Negligible Loss Radiofrequency Metamaterial," Nat Mater., Vol. 10, pp.216-222, March 2011.

[6] S. Hrabar, D. Bonefacic and D. Muha, "Numerical and Experimental Investigation of Basic Properties of Wire Medium-based shortened Horn Antennas," Microw. Opt. Technol. Lett., Vol. 51, pp 2748-2753, November 2009.

[7] A. Tomaz, J. J. Barroso and A. J. F. Orlando, "Control and modificationl of the properties of a horn antenna by a periodic array of metallic wires" (in Portuguese)," $15^{\text {th }}$ SBMO - Brazilian Microwave Symposium and $10^{\text {th }} \mathrm{CBMag}-$ Magnetic Brazilian Congress, João Pessoa, PB, Brazil, 2012.

[8] Advacend Technical Materials, Inc. "Horn Antennas". http://www.atmmicrowave.com/, [Dec. 1, 2012].

[9] J. Baker-Jarvis and M. D. Janezic, "Dielectric and Conductor-loss Characterization and Measurements on Electronic Packaging Materials", Nat. Inst. Standards Technol., Gaithersburg, MD, Tech. Rep. 1520, Jul. 2001.

[10] F. Lemoult, G. Lerosey, J. de Rosny, and M. Fink, "Resonant metalenses for breaking the diffraction barrier", Phys. Rev. Lett., vol. 104, Article ID 203901, May 2010 